\title{
Broadband Ultra-flat Optics With Experimental Efficiencies Exceeding 99\% at Visible Wavelengths
}

\author{
Arturo Burguete-Lopez1 ${ }^{1 *}$, Fedor Getman', Maksim Makarenko', Andrea Fratalocchi ${ }^{1}$ \\ IPRIMALIGHT, Faculty of Electrical Engineering; Applied Mathematics and \\ Computational Science, King Abdullah University of Science and Technology, \\ Thuwal 23955-6900, Saudi Arabia \\ *corresponding author,E-mail:arturo.burguetelopez@kaust.edu.sa
}

\begin{abstract}
We present a platform by which high experimental efficiency (up to 99.2\%), ultraflat (down to $50 \mathrm{~nm}$ ) optics such as polarizer beam splitters, dichroic mirrors and polarization dependent colour filters can be produced in the visible. (C) 2020 The Author(s)
\end{abstract}

\section{Introduction}

Flat-optics hold the promise to replace the traditionally bulky and expensive optical components by highly integrated, versatile flat elements consisting of nanostructured surfaces [1]. While significant progress has been achieved in this field over the past decade, the typical efficiency of flat-optics remains between $20 \%$ and $67 \%$ due to absorption losses in designs that rely on propagation effects to operate [2-4].

In this work we leverage a hidden network of universal approximators, which physically exist in suitably engineered nanostructure layers, to produce high efficiency flat optics capable of furnishing arbitrary optical responses [5]. We employ an inverse design parallel software platform that combines concepts from evolutionary algorithms and the latest generation of convolutional neural networks (CNN's) to determine the optimal geometry of the nanostructures that most closely allows the design to replicates the desired optical response [6]. The devices are capable of simultaneous broadband operation in reflection and transmission and are fabricated using a CMOS compatible process ready for industrial production at inexpensive costs.

\section{Results}

We design, fabricate and characterize a set of seven different devices designed through our platform. In all cases, the devices are manufactured by depositing amorphous silicon on a glass substrate via plasma enhanced chemical vapor deposition (PECVD), patterning, and etching with a chromium mask.

For the purposes of simultaneous polarization control in both reflection and transmission we designed a set of polarizer beam splitters (PBS's) centered at common laser emission lines.

Fig. 1a shows the state of the art in flat-optics PBS's, the efficiency in the visible of existing designs for the best devices lies below $65 \%$ and their operation is limited to transmission. Our designs are capable of simultaneous reflection/transmission operation and present efficiencies above $96 \%$ across the visible and in the near IR.

Fig. 1b shows the efficiency of the produced PBS's, defined as $\eta=\left(T_{\max }-T_{\min }\right) /\left(T_{\max }+T_{\min }\right)$ [7], along with the thickness of each device. An example of the structure of the produced devices, corresponding to the 900nm centered PBS is shown in Fig.1c.

Fig. 1d reports a comparison of the designed and achieved optical response curves for a flat optics dichroic mirror. To the best of the authors knowledge, this is the first implementation of a dichroic mirror using flat optics that has been reported. Fig 1e. shows the concept behind a two sub-pixels flat optics based display technology. Traditional displays, such as liquid crystal displays (LCD's ) employ three filtering elements per pixel to produce colour images by manipulating the intensity of the light that passes through each of them. We propose a pixel that employs only two sub-elements. Each element acts as a polarization dependent colour filter, when the polarization of the impinging light on an element is rotated, the element changes between blue and red or between blue and green depending on the design. Coupled with a variable intensity backlight, this scheme allows for the creation of a colour display that can pack 33\% more pixels for a given area and consumes 33\% less energy per pixel compared to a traditional display. The $1931 \mathrm{CIE}$ colorspace coverage of a first design is shown at the bottom of the panel. Each bold black line represents the gamut achieved by the individual structures as the polarization is rotated. Renders of the designs found to achieve this gamut coverage are shown to the right of this figure. 

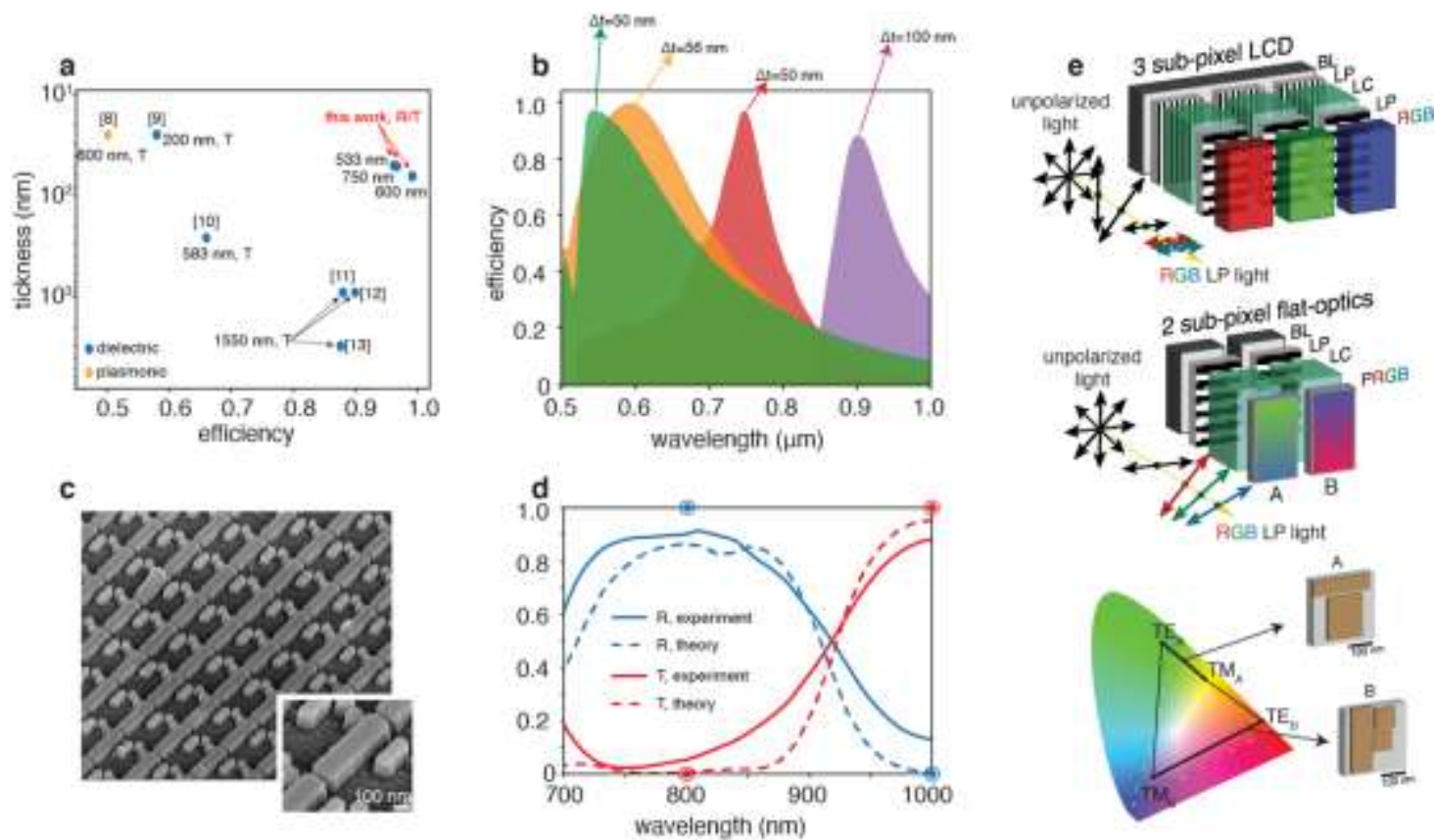

Figure 1. a state of the art in PBS's. b Experimental efficiency and thickness of the produced PBS's. c SEM image of the PBS centered at 900nm. d Optical response, theoretical and experimental, of the dichroic mirror design. e Comparison of traditional display technologies with the proposed approach, achieved design gamut.

\section{References}

1. Nanfang Yu and Federico Capasso, "Flat optics with designer metasurfaces," Nat. Mater. 13, 139--150 (2014).

2. Stanislav B. Glybovski, Sergei A. Tretyakov, Pavel A. Belov, Yuri S. Kivshar, and Constantin R. Simovski, "Metasurfaces: From microwaves to visible," Phys. Rep. 634, 1--72 (2016).

3. Z. Guo, H. Xu, K. Guo, F. Shen, H. Zhou, Q. Zhou, J. Gao, and Z. Yin, "High-efficiency visible transmitting polarizations devices based on the GaN metasurface," Nanomaterials 8, 333 (2018).

4. Shane Colburn, Alan Zhan, Elyas Bayati, James Whitehead, Albert Ryou, Luocheng Huang, and Arka Majumdar, "Broadband transparent and CMOS-compatible flat optics with silicon nitride metasurfaces [Invited]," Opt. Mater. Express 8, 2330 (2018).

5. A. Burguete-Lopez, M. Makarenko, F. Getman, and Andrea Fratalocchi, "Artificial intelligence inverse design of ultra-flat meta-optics with experimental efficiencies exceeding $99 \%$ in the visible," in Society of Photo-Optical Instrumentation Engineers (SPIE) Photonics West Conference Series (2020), Vol. 1129933.

6. M. Makarenko, A. Burguete-Lopez, F. Getman, and A. Fratalocchi, "Generalized Maxwell projections for multi-mode network Photonics," ArXiv200208121 Phys. (2020).

7. M. Bass and V. N. Mahajan, Handbook of Optics. Volume I, Geometrical and Physical Optics, Polarized Light, Components and Intruments, 3rd ed. (McGraw-Hill, 2010).

8. V. Pelletier, K. Asakawa, M. Wu, D. H. Adamson, R. A. Register, and P. M. Chaikin, "Aluminum nanowire polarizing grids: Fabrication and analysis," Appl. Phys. Lett. 88, 211114 (2006).

9. Y.-R. Hong, K. Asakawa, D. H. Adamson, P. M. Chaikin, and R. A. Register, "Silicon nanowire grid polarizer for very deep ultraviolet fabricated from a shear-aligned diblock copolymer template," Opt. Lett. 32, 3125-3127 (2007).

10. J. Li, C. Liu, T. Wu, Y. Liu, Y. Wang, Z. Yu, H. Ye, and L. Yu, "Efficient Polarization Beam Splitter Based on All-Dielectric Metasurface in Visible Region," Nanoscale Res. Lett. 14, 34 (2019).

11. Z. Guo, L. Zhu, K. Guo, F. Shen, and Z. Yin, "High-Order Dielectric Metasurfaces for High-Efficiency Polarization Beam Splitters and Optical Vortex Generators," Nanoscale Res. Lett. 12, 512 (2017).

12. Z. Guo, L. Zhu, F. Shen, H. Zhou, and R. Gao, "Dielectric metasurface based high-efficiency polarization splitters," RSC Adv. 7, 9872-9879 (2017).

13. B. Wang, C. Zhou, S. Wang, and J. Feng, "Polarizing beam splitter of a deep-etched fused-silica grating," Opt. Lett. 32, 1299-1301 (2007).

Acknowledgements This research received funding from KAUST (AWARD OSR-2016-CRG5-2995). Parallel simulations are performed on KAUST's Shaheen supercomputer. 